Informe especial

\title{
Os efeitos do chumbo sobre o organismo humano e seu significado para a saúde
}

\author{
Fátima Ramos Moreira ${ }^{1}$ e Josino Costa Moreira ${ }^{1}$
}

Como citar Moreira FR, Moreira JC. Os efeitos do chumbo sobre o organismo humano e seu significado para a saúde. Rev Panam Salud Publica. 2004;15(2):119-29.

RESUMO Objetivo. Fazer um levantamento bibliográfico acerca dos riscos associados à exposição ao chumbo e seus compostos, especialmente nas populações expostas ocupacionalmente e nas crianças. Fonte dos dados. Pesquisa do período de 1988 a 2002 nas bases de dados PubMed e Literatura Latino-Americana e do Caribe em Ciências da Saúde (LILACS); página da agência de controle de substâncias tóxicas e doenças do governo dos Estados Unidos (Agency for Toxic Substances and Disease Registry) na Internet; acervo bibliográfico do Laboratório de Toxicologia do Centro de Estudos da Saúde do Trabalhador e Ecologia Humana da Escola Nacional de Saúde Pública, Brasil.

Conclusões. Os estudos científicos sobre a toxicologia do chumbo são desenvolvidos há mais de um século. Contudo, ainda são insuficientes as informações sobre os mecanismos de ação que originam os efeitos tóxicos desse metal. Os resultados da exposição ao chumbo sobre os ossos, os sistemas nervoso central e cardiovascular, os rins e o fígado devem ser estudados com maior profundidade, bem como os efeitos sobre a reprodução masculina e feminina, o sistema endócrino e a formação do feto. Também é essencial esclarecer se o chumbo tem efeitos teratogênicos e carcinogênicos em seres humanos.

Palavras-chave Chumbo, metais pesados/toxicidade.

Os metais pesados podem danificar toda e qualquer atividade biológica. Por isso há, teoricamente, tantos tipos de respostas biológicas a esses metais quantos forem os tipos de atividade biológica. Todavia, o acesso variado aos componentes biológicos faz com que certos tipos de respostas predomi-

\footnotetext{
1 Fundação Oswaldo Cruz, Escola Nacional de Saúde Pública (ENSP), Centro de Estudos da Saúde do Trabalhador e Ecologia Humana (CES$\mathrm{TEH})$, Rio de Janeiro (RJ), Brasil. Correspondência e pedidos de separatas devem ser enviados a Fátima Ramos Moreira no seguinte endereço: Rua Leopoldo Bulhões 1480, CEP 21041-210, Manguinhos, RJ, Brasil. Fone: +55-21-2598-2819/25982820; fax: +55-21-2270-3219; e-mail: fmoreira@ensp. fiocruz.br
}

nem. Por exemplo, todos os sistemas enzimáticos são potencialmente suscetíveis aos metais pesados. Por outro lado, nos organismos vivos, o acesso dos metais pesados pode ser limitado pelas estruturas anatômicas; além disso, os sítios ligantes inertes podem competir pelo íon metálico. Por essas razões, freqüentemente existem consideráveis diferenças de sensibilidade entre diferentes órgãos e tecidos, assim como na ação observada entre experimentos in vivo e in vitro, entre espécies e entre respostas típicas de envenenamento clínico (1).

A toxicidade do chumbo gera desde efeitos claros, ou clínicos, até efeitos sutis, ou bioquímicos. Estes últimos envolvem vários sistemas de órgãos e atividades bioquímicas. Nas crianças, os efeitos críticos atingem o sistema nervoso, enquanto que nos adultos com exposição ocupacional excessiva, ou mesmo acidental, os cuidados são com a neuropatia periférica e a nefropatia crônica. Em situações raras, os efeitos sobre a síntese da heme proporcionam indicadores de exposição ao chumbo na ausência de conseqüências quimicamente perceptíveis. Também os sistemas gastrintestinal e reprodutivo são alvo da intoxicação pelo chumbo (2).

Nos últimos anos, atenção especial tem sido dada aos estudos epidemiológicos direcionados para os possíveis 
efeitos neurotóxicos do chumbo nas crianças, especialmente naquelas com desvios de comportamento. Na população adulta, esforços consideráveis têm sido dispensados no sentido de avaliar os efeitos cardiovasculares do chumbo e o seu envolvimento na hipertensão. Além disso, diversos estudos têm contribuído para a compreensão dos efeitos bioquímicos desse metal, podendo facilitar o reconhecimento precoce de alterações significativas e para a suavização de conseqüências potencialmente adversas. Tais estudos indicam que os efeitos do chumbo são os mesmos para a população em geral e para a população exposta ocupacionalmente. Entretanto, é importante distinguir entre adultos e crianças, devido à diferença de suscetibilidade entre esses dois grupos (3).

Em função da ausência de publicações sobre o tópico em português, o objetivo deste artigo foi fazer um levantamento bibliográfico acerca dos riscos para a saúde humana associados com a exposição ao chumbo e seus compostos, especialmente nas populações expostas ocupacionalmente e nas crianças. $\mathrm{O}$ foco principal foi a busca de informações recentes sobre efeitos não confirmados atribuídos ao chumbo.

\section{FONTE DOS DADOS}

Foram pesquisadas as bases de dados PubMed e Literatura LatinoAmericana e do Caribe em Ciências da Saúde (LILACS) no período de 1988 a 2002. Também foi pesquisada a página da agência de controle de substâncias tóxicas e doenças do governo dos Estados Unidos (Agency for Toxic Substances and Disease Registry, ATSDR) na Internet. $\mathrm{O}$ acervo bibliográfico do Laboratório de Toxicologia do Centro de Estudos da Saúde do Trabalhador e Ecologia Humana, pertencente à Escola Nacional de Saúde Pública, unidade da Fundação Oswaldo Cruz, órgão do Ministério da Saúde do Brasil, também foi de grande valor nesta investigação.

Estudos que descrevessem diretamente os efeitos do chumbo sobre órgãos e sistemas foram selecionados a partir das palavras-chave "chumbo", "efeitos" e nome de cada um dos órgãos ou sistemas sobre os quais esse metal possivelmente exerceria um efeito adverso.

\section{TOXICOLOGIA GERAL}

O chumbo é um elemento tóxico não essencial que se acumula no organismo. Na sua interação com a matéria viva, o chumbo apresenta tanto características comuns a outros metais pesados quanto algumas peculiaridades. Como esse metal afeta virtualmente todos os órgãos e sistemas do organismo, os mecanismos de toxicidade propostos envolvem processos bioquímicos fundamentais, que incluem a habilidade do chumbo de inibir ou imitar a ação do cálcio e de interagir com proteínas. Em níveis de exposição moderada (ambiental e ocupacional), um importante aspecto dos efeitos tóxicos do chumbo é a reversibilidade das mudanças bioquímicas e funcionais induzidas.

A toxicidade do chumbo resulta, principalmente, de sua interferência no funcionamento das membranas celulares e enzimas, formando complexos estáveis com ligantes contendo enxofre, fósforo, nitrogênio ou oxigênio (grupamentos $\left.-\mathrm{SH},-\mathrm{H}_{2} \mathrm{PO}_{3},-\mathrm{NH}_{2},-\mathrm{OH}\right)$, que funcionam como doadores de elétrons $(1,4)$. As interações bioquímicas do chumbo com grupamentos -SH são consideradas de grande significado toxicológico, visto que, se tal interação ocorrer em uma enzima, sua atividade pode ser alterada e resultar em efeitos tóxicos. O chumbo também tem alta afinidade com as aminas e os aminoácidos simples. A estabilidade dos complexos de chumbo aumenta com o número crescente de sítios ligantes e com espaçamentos ótimos, como no caso dos grupamentos sulfidrilas vicinais. A habilidade do chumbo de mimetizar o cálcio na ativação da calmodulina envolve ligação com grupos carboxilas, enquanto que os grupos sulfidrilas estão relacionados com a ativação da proteína quinase C. Portanto, os mecanismos de mimetismo do cálcio e de ligação com proteínas algumas vezes se sobrepõem. Em geral, essas ligações dos íons $\mathrm{Pb}$ com o material bioquímico são fortes, porém inespecíficas com respeito aos efeitos do chumbo, e até agora não têm sido aplicadas no monitoramento biológico (1, 4-6).

\section{EFEITOS BIOLÓGICOS}

Os efeitos biológicos do chumbo são os mesmos qualquer que seja a rota de entrada (inalação ou ingestão), uma vez que há interferência no funcionamento normal da célula e em inúmeros processos fisiológicos $(4,7)$. As maiores concentrações de chumbo são encontradas nos ossos, porém os primeiros efeitos adversos da exposição ao metal não são aí observados; inclusive, são limitadas as informações com respeito ao efeito e potenciais mecanismos de ação do chumbo sobre os ossos (4). Adachi et al. (8) encontraram maior conteúdo de chumbo no osso cortical de pacientes com doença de Paget, com osteoporose e sob diálise do que em controles. Entretanto, os níveis do metal no osso trabecular foram mais baixos nos pacientes com doença de Paget ou osteíte fibrosa. Assim, os autores não puderam confirmar a existência de uma associação entre o chumbo e a doença no osso.

O sistema nervoso, a medula óssea e os rins são sítios críticos na exposição ao chumbo, enquanto que os distúrbios na função do sistema nervoso e os desvios na síntese da heme são considerados como efeitos tóxicos críticos (9). Os efeitos sobre o sistema nervoso ocorrem sempre que os níveis de chumbo no sangue ( $\mathrm{Pb}-\mathrm{S}$ ) forem da mesma ordem de grandeza daqueles que alteram a síntese da heme. Por isso, os distúrbios na biossíntese da heme servem como "indicador metabólico" para a detecção precoce de exposição perigosa ao chumbo antes do aparecimento de sintomas clínicos (6).

\section{Efeitos neurológicos}

O conjunto de órgãos mais sensível ao envenenamento por chumbo é o sistema nervoso, sendo que a encefalo- 
patia é um dos mais sérios desvios tóxicos induzidos pelo chumbo em crianças e adultos. Além da ausência de um limite preciso, a toxicidade do chumbo na infância pode ter efeitos permanentes, tais como menor quociente de inteligência e deficiência cognitiva. Durante o desenvolvimento de uma criança, o sistema nervoso pode ser afetado adversamente por valores de $\mathrm{Pb}-\mathrm{S}$ menores do que $10 \mu \mathrm{g} . \mathrm{L}^{-1}$, níveis antes considerados seguros (10, 11). Nos adultos, o sistema nervoso central também é afetado por concentrações relativamente baixas ( $\mathrm{Pb}-\mathrm{S}$ em torno de $40 \mu \mathrm{g} . \mathrm{dL}^{-1}$ ). Os danos sobre o sistema nervoso periférico, primeiramente motor, são observados principalmente nos adultos $(1,3,5,7)$.

A encefalopatia causada pelo chumbo ocorre nas formas aguda e crônica. O curso clínico da encefalopatia aguda pelo chumbo varia, dependendo da idade e da condição geral do paciente, da quantidade absorvida, do tempo de exposição e de certos fatores concomitantes, como o alcoolismo crônico. A relação dose-resposta para as desordens do sistema nervoso central não é bem conhecida. Encefalopatia aguda se desenvolve somente após doses maciças e é rara quando os níveis de $\mathrm{Pb}-\mathrm{S}$ estão abaixo de 100 $\mu \mathrm{g} . \mathrm{dL}^{-1}(1,3,5)$.

A encefalopatia crônica pode ser um estado residual após a encefalopatia aguda originada por esse metal, mas também pode resultar de uma exposição prolongada ao chumbo (12). As crianças são mais suscetíveis do que os adultos aos efeitos da encefalopatia sobre o sistema nervoso central. A exposição pode começar ainda no útero, caso a mãe tenha chumbo em seu organismo, e aumentar após o nascimento, através de inúmeras fontes (4). A intoxicação pediátrica com chumbo tem efeitos comportamentais e psicológicos que, juntamente com a disfunção da percepção sensório-motora fina e com alterações na eletroencefalografia, estão relacionados com uma dose recebida no passado $(1,6)$. De acordo com alguns estudos, tais efeitos podem também ser induzidos pela exposição prolongada, em nível moderado, em idade precoce (13). Os meca- nismos dos efeitos neurotóxicos do chumbo não são bem conhecidos, porém existem indicações de distúrbios no metabolismo do carboidrato, síntese anormal de nucleotídeos, inibição da respiração celular, bloqueio dos grupamentos - $\mathrm{SH}$ neuronais e mudanças nos níveis de ácido neuramínico e RNA (3).

Em adultos, alguns estudos indicam que os efeitos claros da neurotoxicidade aparecem com níveis $\mathrm{Pb}-\mathrm{S}$ de 40 a $60 \mu \mathrm{g} . \mathrm{dL}^{-1}$, concentração na qual também se fazem presentes outros sinais e sintomas claros de intoxicação por chumbo, tais como as queixas gastrintestinais. Diversos testes neurocomportamentais também mostraram efeitos com concentrações de chumbo no sangue de aproximadamente $30 \mu \mathrm{g} \cdot \mathrm{dL}^{-1}$ (4). No entanto, Osterberg et al. (14) estudaram 38 trabalhadores expostos ao chumbo e concluíram que um $\mathrm{Pb}-\mathrm{S}$ de $37,3 \mu \mathrm{g} \cdot \mathrm{dL}^{-1}$ não estava associado a efeitos comportamentais adversos, e que uma exposição prolongada (13 anos, em média), com $\mathrm{Pb}-\mathrm{S}$ em torno de $41,4 \mu \mathrm{g} \cdot \mathrm{dL}^{-1}$, não estava associada à disfunção permanente do cérebro.

Hanninen et al. (15) estudaram 54 trabalhadores de uma fábrica de baterias e concluíram que as alterações neuropsicológicas encontradas nos sujeitos com exposições passadas altas e presentes baixas indicam que concentrações de $\mathrm{Pb}-\mathrm{S}$ na faixa de 51,8 a $101,4 \mu \mathrm{g} . \mathrm{dL}^{-1}$ podem causar danos prolongados, ou mesmo permanentes, na função do sistema nervoso central. Já Schwartz et al. (16) relataram que a função cognitiva pode diminuir progressivamente devido a exposições ocupacionais passadas ao chumbo, após avaliação dos testes neurocomportamentais realizados em adultos com exposição passada ao metal. A avaliação psicológica de trabalhadores de fábricas de baterias com níveis elevados de $\mathrm{Pb}-\mathrm{S}$ mostrou que as deficiências funcionais do sistema nervoso central não estavam relacionadas com o tempo de exposição, e sim com o PbS (17). Nordberg et al. (18) não encontraram correlação entre a concentração de $\mathrm{Pb}-\mathrm{S}$ e a função cognitiva em uma população de idosos (idade média de 88,4 anos) não expostos.
A paralisia do sistema nervoso periférico é caracterizada pelo envolvimento seletivo dos nervos motores (19). Também afeta os músculos extensores unilateralmente, sendo típica a queda do pulso do braço direito. A maioria dos dados sobre humanos sugere que a lesão é neurogênica e periférica, geralmente referida como neuropatia (7). Não são conhecidos os níveis em que as primeiras anormalidades neurofisiológicas aparecem, mas a experiência escandinava sugere que alterações leves já podem aparecer em concentrações entre 40 e $70 \mu \mathrm{g} \cdot \mathrm{dL}^{-1}$ (1).

Os efeitos sobre o nervo ótico e o sistema auditivo também têm sido atribuídos à exposição ao chumbo. Em crianças, um aumento de 6,2 para 18,6 $\mu \mathrm{g} . \mathrm{dL}^{-1}$ no $\mathrm{Pb}-\mathrm{S}$ corresponde a uma diminuição na audição de $2 \mathrm{~dB}$ em todas as freqüências. Efeitos leves sobre o sistema nervoso autônomo foram registrados num grupo de trabalhadores com média de $\mathrm{Pb}-\mathrm{S}$ de $33,1 \mu \mathrm{g} \cdot \mathrm{dL}^{-1}$ (5).

Os experimentos de Murata et al. (20) confirmam as observações de outros estudos, pois sugerem que o chumbo afeta não somente o nervo periférico, mas também as funções nervosas central e autônoma em nível subclínico. Também sugerem que o zinco pode antagonizar os efeitos neurotóxicos do chumbo. Testes neurofisiológicos foram realizados em 41 homens expostos ocupacionalmente, e os resultados indicaram que a exposição ao chumbo tem um maior efeito sobre a função de condução no sistema nervoso periférico do que no central, nos percursos sensorial e auditivo, e inversamente no visual (21). Já Murata et al. (22) observaram que a função nervosa autônoma é mais suscetível ao chumbo do que as funções nervosas visuais e auditivas; o chumbo afeta mais fortemente a atividade simpática do que a parassimpática. Araki et al. (23) fizeram uma revisão das pesquisas voltadas aos efeitos neurofisiológicos subclínicos do chumbo em trabalhadores ocupacionalmente expostos. Os dados disponíveis sugeriram que tais efeitos ocorrem a uma concentração de $\mathrm{Pb}-\mathrm{S}$ na faixa de 30 a $50 \mu$ g.dL ${ }^{-1}$. A avaliação das funções neurofisiológicas de trabalhadores com baixa exposição ao 
chumbo forneceu medidas sensíveis dos efeitos desse metal em adultos expostos ocupacionalmente (24).

\section{Efeitos hematológicos}

A anemia é uma descoberta extraordinária no envenenamento por chumbo, não estando necessariamente associada com deficiência de ferro. Geralmente, é de leve a moderada em adultos (os valores de hemoglobina variam de 8 a $12 \mathrm{~g} / 100 \mathrm{~mL}^{-1}$ ) e, algumas vezes, é severa em crianças (1). Os desvios hematológicos que levam à anemia pelo chumbo são considerados como resultado de sua ação tóxica sobre as células vermelhas e eritropoiéticas na medula óssea. Esses efeitos incluem inibição da síntese da hemoglobina $(\mathrm{Hb})$ e diminuição do tempo de vida dos eritrócitos circulantes, resultando na estimulação da eritropoese. Entretanto, a anemia não é uma manifestação precoce do envenenamento por chumbo, sendo rara sem outros efeitos detectáveis, e só é evidente quando o nível de $\mathrm{Pb}-\mathrm{S}$ é significativamente elevado por períodos prolongados $(3,4,7)$.

Hu et al. (25) concluíram que os níveis de chumbo encontrados na patela estão associados com níveis reduzidos de $\mathrm{Hb}$ e hematócrito, a despeito da presença de baixos níveis de $\mathrm{Pb}-\mathrm{S}$, podendo refletir um efeito subclínico dos estoques de $\mathrm{Pb}$ no osso sobre a hematopoese. Uma pesquisa com crianças de 5 a 14 anos concluiu que a morfologia e a função dos eritrócitos poderiam ser parâmetros sensíveis da toxicidade do chumbo em doses baixas (26).

Serwint et al. (27) estudaram os níveis de ferro em crianças de 11 a 33 meses e concluíram que esse parâmetro não diferia entre as crianças expostas ao chumbo ( $\mathrm{Pb}-\mathrm{S}$ de 20 a $\left.44 \mu \mathrm{g} . \mathrm{dL}^{-1}\right)$ e os controles $\left(\mathrm{Pb}-\mathrm{S}<10 \mu \mathrm{g} \cdot \mathrm{dL}^{-1}\right)$. Wright (28) relata que diversos estudos demonstraram que os animais deficientes em ferro têm a absorção do chumbo aumentada, porém o tratamento com suplementos de ferro diminui a excreção do chumbo, um fator que poderia exacerbar a toxicidade do chumbo enquanto suavizaria os efeitos da deficiência de ferro.
Os dados encontrados por Osterode et al. (29) sugerem novos aspectos da anemia induzida pelo chumbo além do período reduzido de vida dos eritrócitos e inibição da síntese da $\mathrm{Hb}$, normalmente reconhecidos. Dois mecanismos adicionais devem ser considerados: a redução das células progenitoras eritrocitárias (BFU-E) e a produção renal inapropriada de eritropoietina (EPO) na presença de exposição severa ao chumbo, que levaria a uma maturação inadequada das células BFU-E. Counter et al. (30) observaram uma correlação inversa significativa entre níveis de chumbo e $\mathrm{Hb}$ no sangue de 88 crianças expostas, e concluíram que a anemia era provavelmente induzida pelo chumbo. Suplido e Ong (31) observaram que $90 \%$ dos trabalhadores de uma reformadora de baterias com $\mathrm{Pb}-\mathrm{S}>40 \mu \mathrm{g} \cdot \mathrm{dL}^{-1}$ eram anêmicos $\left(\mathrm{Hb}<13\right.$ g.dL ${ }^{-1}$ para homens e $<11,5$ g.dL ${ }^{-1}$ para mulheres). A regressão linear mostrou uma correlação entre o nível de $\mathrm{Hb}$ e log (Pb-S), porém não houve uma relação significativa entre a anemia e o $\mathrm{Pb}-\mathrm{S}$ em crianças que moravam nas redondezas da reformadora.

O chumbo inibe a capacidade do organismo de produzir $\mathrm{Hb}$, afetando várias reações enzimáticas, críticas para a síntese da heme. As atividades de três enzimas - 5-aminolevulinato desidratase, coproporfirinogênio oxidase e ferroquelatase - são inibidas pelo chumbo. Isso enfraquece a síntese da heme e desencoraja a síntese da 5-aminolevulinato sintetase, enzima inicial e limitante da taxa da biossíntese da heme, e da coproporfirinogênio descarboxilase. Em conseqüência, há maior produção e excreção dos precursores 5-aminolevulinato sintetase e coproporfirina, com aumento na protoporfirina circulante, geralmente ligada ao zinco. Nas células vermelhas, a síntese diminuída de mono-oxigenases (citocromos P-450) compromete a oxidação de drogas e o chumbo se liga à $\mathrm{Hb}$. A ferroquelatase, que cataliza a inserção de ferro na protoporfirina IX, é completamente sensível ao chumbo. Entretanto, a inibição desta enzima é um fator limitante da taxa para a síntese da $\mathrm{Hb}$, já que a protoporfirina IX se acumula nos eritrócitos, constituindo cerca de $95 \%$ das porfirinas não ligadas ao ferro nas células vermelhas. Assim, uma diminuição na atividade da ferroquelatase resulta em aumento do substrato, protoporfirina eritrocitária, nas células vermelhas $(1,3,4,6)$.

Vahter et al. (32) pesquisaram o sangue de crianças expostas (mediana $\mathrm{Pb}$ S: $60,0 \mu \mathrm{g} \cdot \mathrm{dL}^{-1}$ ) e os resultados mostraram um decréscimo significativo e um aumento marcante nas concentrações de $\mathrm{Hb}$ e protoporfirina, respectivamente, com elevação do nível de $\mathrm{Pb}-\mathrm{S}$, indicando um efeito sobre a síntese da heme. No entanto, Froom et al. (33) examinaram a correlação entre os níveis de $\mathrm{Hb}, \mathrm{Pb}-\mathrm{S}$ e zinco protoporfirina em 961 amostras de sangue (níveis $>60 \mu \mathrm{g} \cdot \mathrm{dL}^{-1}$ em $14 \%$ das amostras) de 94 trabalhadores expostos e as concentrações de $\mathrm{Hb}$ não se correlacionaram com o nível de chumbo ou zinco protoporfirina no sangue. Assim, os autores concluíram que a anemia encontrada em pessoas com nível de $\mathrm{Pb}-\mathrm{S}$ de até $80 \mu$ g.dL $\mathrm{dL}^{-1}$ deveria ser atribuída exclusivamente ao chumbo, após todas as outras causas terem sido excluídas.

Os efeitos hematológicos do chumbo são os únicos para os quais as relações dose-resposta foram estabelecidas com acurácia e, por isso mesmo, pressupõe-se que a concentração de $\mathrm{Pb}-\mathrm{S}$ represente a dose ao qual o indivíduo foi exposto. Dessa forma, várias das alterações hematológicas servem como testes para o diagnóstico de absorção excessiva: os efeitos sobre a síntese da heme fornecem indicadores bioquímicos de exposição ao chumbo na ausência de marcadores quimicamente detectáveis $(2,7)$.

\section{Efeitos endocrinológicos}

O chumbo parece interferir na conversão da vitamina $D$ em 1,25-dihidroxivitamina D. Nas crianças, as concentrações de 1,25-dihidroxivitamina D no soro são usadas como um indicador dos efeitos do chumbo sobre o sistema de enzimas que medeiam a hidroxilação inicial. Entretanto, outros fatores, tais como dieta, necessidades fisiológicas de cálcio e fósforo e níveis de hormônios 
calciotrópicos, tais como o paratireóide, podem regular a produção e concentração da 1,25-dihidroxivitamina D no organismo $(3,4)$.

Vários estudos mostram a existência de uma correlação inversa forte entre os níveis de chumbo no sangue e de 1,25-dihidroxivitamina D no soro. Entretanto, os dados epidemiológicos permitiram concluir que os efeitos do chumbo sobre o metabolismo da vitamina D, observados em alguns estudos, somente eram evidentes em crianças com deficiência nutricional crônica e níveis de chumbo em sangue elevados por longo tempo $(4,7)$.

Koo et al. (34) estudaram, por até 33 meses, 105 crianças com conhecida exposição ao chumbo. Esses autores concluíram que não havia alterações significativas no metabolismo da vitamina D, homeostase de cálcio e fósforo e conteúdo mineral ósseo nas crianças com estado nutricional adequado e exposição de baixa a moderada ao chumbo. Diversos estudos concluíram que a interferência do chumbo na síntese da heme pode ser a base para os efeitos sobre o metabolismo da vitamina D. Além disso, aparentemente, as alterações iniciais causadas pelo chumbo sobre o metabolismo renal da vitamina $\mathrm{D}$ são semelhantes àquelas causadas pela acumulação da protoporfirina eritrocitária (4). Como o conjunto vitamina D-glândulas endócrinas é, em grande parte, responsável pela manutenção da homeostase do cálcio extra e intra-celular, é razoável concluir que a interferência do chumbo na produção renal de 1,25dihidroxivitamina $\mathrm{D}$ terá um impacto sobre processos fundamentais por todo o corpo, podendo provavelmente prejudicar o crescimento e a maturação da célula e o desenvolvimento de dentes e ossos, entre outros $(4,7)$.

Em seres humanos, há indicações de que a exposição ao chumbo cause prejuízos endócrinos aos eixos hipotálamo-pituitária-tireóide/supra-renal. No entanto, os efeitos relatados não são consistentes e, em outros estudos, não são sequer encontrados. As observações descritas podem indicar danos na função da tireóide pelo impedimento da entrada de iodo, prova- velmente através da interferência no eixo pituitária-supra-renal. Também as funções das glândulas supra-renal e pituitária podem ser afetadas. Porém, os resultados positivos encontrados devem ser vistos com cautela, uma vez que os estudos não citam se as variáveis de confundimento foram controladas $(1,4,5)$.

Ao estudar 58 homens expostos ocupacionalmente ao chumbo por 156,9 \pm 122,7 meses, Singh et al. (35) concluíram que níveis de $\mathrm{Pb}-\mathrm{S} \geq 50 \mu \mathrm{g} \cdot \mathrm{dL}^{-1}$ podem acentuar a liberação de hormônio estimulante da tireóide (TSH) da pituitária sem que haja qualquer alteração significativa nos níveis circulantes de T3 e T4. Erfurth et al. (36) também estudaram os efeitos do chumbo sobre o sistema endócrino de 77 trabalhadores de uma fundição de chumbo. Os resultados mostraram que uma exposição moderada ao chumbo estava associada somente com pequenas alterações na função endócrina masculina, afetando principalmente o eixo hipotálamo-pituitária.

\section{Efeitos sobre o crescimento}

Diversas pesquisas têm sugerido que o crescimento físico e a estatura das crianças podem ser reduzidos pela exposição ao chumbo. Nos Estados Unidos, um levantamento nacional realizado de 1976 a 1980 com 2695 crianças com idade igual ou menor do que 7 anos forneceu evidências de uma associação entre níveis crescentes de chumbo no sangue e redução no peso, altura e circunferência de tórax, após ajuste para idade, raça, sexo e fatores nutricionais $(3,4)$. Uma edição posterior do mesmo levantamento, com dados coletados de 1988 a 1994 entre 4391 crianças de 1 e 7 anos, concluiu que as associações negativas significativas entre a concentração de $\mathrm{Pb}-\mathrm{S}$ e a estatura e circunferência da cabeça eram semelhantes em grandeza àquelas relatadas no levantamento anterior (37).

A exposição ao chumbo também foi associada com baixa estatura em crianças nos estudos de Berglund et al. (38). Vivoli et al. (39) avaliaram a relação entre o crescimento somático e a exposição ao chumbo em 418 adolescentes e seus dados sugerem que, mesmo para baixa exposição ao chumbo, este metal pode afetar o crescimento em estatura (13) e os níveis de gonadotropinas. Já os resultados encontrados por Hicks et al. (40) demonstram importantes efeitos reguladores do chumbo sobre os condrócitos em cultura de células, e sugerem um efeito inibidor sobre o processo de formação endocondrial do osso, sendo que a placa de crescimento pode ser um dos principais tecidos alvo a serem considerados em termos dos efeitos adversos da exposição crônica ao chumbo sobre o desenvolvimento dos ossos (41).

\section{Efeitos renais}

A exposição excessiva e prolongada ao chumbo pode causar doença renal progressiva e irreversível. A nefropatia por chumbo é caracterizada por uma redução gradual da função renal e é freqüentemente acompanhada por hipertensão $(1,3)$. Os efeitos tóxicos do chumbo sobre os rins ocorrem na presença de níveis relativamente altos de $\mathrm{Pb}-\mathrm{S}$ e se dividem principalmente em disfunção tubular renal reversível e nefropatia intersticial irreversível.

A disfunção reversível ocorre, na maior parte, em crianças sob exposição aguda - basicamente por via oral ao chumbo (e algumas vezes em trabalhadores expostos). Essa disfunção está geralmente associada a efeitos conhecidos sobre o sistema nervoso central. As características da nefropatia aguda incluem corpos de inclusão nuclear, alterações fisiológicas na mitocôndria e citomegalia das células epiteliais dos túbulos proximais. A nefropatia irreversível, um efeito direto da exposição crônica sobre os rins, é caracterizada por esclerose vascular, atrofia ou hiperplasia da célula tubular, fibrose intersticial progressiva, nenhum ou poucos corpos de inclusão e esclerose glomerular. A forma crônica é descrita principalmente em trabalhadores expostos, cuja exposição primária é por inalação (42). Nos estágios iniciais dessa exposição excessiva 
aguda, as alterações morfológicas e funcionais nos rins estão limitadas aos túbulos renais e são mais pronunciadas nas células tubulares proximais, cujos danos se manifestam por reabsorção reduzida de aminoácidos, glicose, fosfato e ácido cítrico. Em casos severos, podem ocorrer hiperaminoacidúria, glicosúria e hipofosfatemia combinada com hiperfosfatúria (síndrome parecida com a de Fanconi) (1, 3-5, 7).

Loghman-Adham (43) afirma que a relação entre a disfunção renal detectada por testes sensíveis, tais como creatinina no soro, excreção urinária de proteínas de baixo peso molecular e enzimas lisossomais, e o desenvolvimento futuro de doença renal crônica permanece incerta para exposição a níveis baixos de chumbo. $\mathrm{O}$ diagnóstico de função alterada ou doença renal induzida por chumbo é difícil, uma vez que não há indicadores específicos; os níveis de uréia no sangue e de creatinina no soro se tornam elevados somente depois da perda de dois terços da função renal. Esses efeitos sobre a taxa de filtração glomerular e excreção de ácido úrico ocorrem em grupos de trabalhadores com média de $\mathrm{Pb}-\mathrm{S}$ de mais de 52 a $72 \mu \mathrm{g} . \mathrm{dL}^{-1}(3,5)$. Os estudos já realizados fornecem evidências de que a nefropatia crônica está associada com níveis de $\mathrm{Pb}-\mathrm{S}$ variando de 40 a mais de $100 \mu \mathrm{g} \cdot \mathrm{dL}^{-1}$, enquanto que, em crianças, a nefropatia só ocorre com concentrações acima de $80 \mu \mathrm{g} \cdot \mathrm{dL}^{-1}$, geralmente excedendo $120 \mu \mathrm{g} \cdot \mathrm{dL}^{-1}(4)$.

Há também evidências de uma associação entre a exposição ao chumbo e a hipertensão (44), um efeito que pode ser mediado através dos mecanismos renais. $\mathrm{O}$ chumbo parece afetar o metabolismo da vitamina $\mathrm{D}$ nas células do túbulo renal, de modo que os níveis circulantes do hormônio da vitamina D são reduzidos (1, 2, 4, 45).

A gota saturnínica é uma conseqüência da função tubular reduzida (46), pois o chumbo interfere na excreção dos sais de ácido úrico. Conseqüentemente, embora suas taxas de formação sejam normais, o nível do ácido úrico se torna elevado no sangue, e seus cristais são depositados nas articulações, como na gota comum. Assim, a gota pode se desenvolver como resultado da hiperuricemia induzida pelo chumbo, com a redução seletiva da excreção fracionada do ácido úrico, antes de um declínio na depuração da creatinina $(2,5,7)$. A gota saturnínica difere da comum, uma vez que ambos os sexos são igualmente afetados e a função renal é sempre danificada (1).

\section{Efeitos sobre a reprodução e o desenvolvimento}

É geralmente aceita a noção de que o chumbo causa efeitos adversos sobre o sistema reprodutor masculino e feminino; porém, as evidências são principalmente qualitativas, e não foram estabelecidas relações de dose-resposta. Uma maior freqüência de abortos e partos de natimortos entre mulheres que trabalham com o chumbo foi relatada no início do século $X X$. Embora os dados sobre os níveis de exposição sejam incompletos, esses efeitos provavelmente resultaram de uma exposição maior ao chumbo do que aquela normalmente encontrada em indústrias de chumbo (7). A associação entre a exposição ocupacional severa ao chumbo e os abortos espontâneos levou à exclusão de mulheres das ocupações com alta exposição. No entanto, os estudos iniciais sofriam de deficiências metodológicas (4). Da mesma forma, são escassos os dados confiáveis sobre a relação de doseefeito na reprodução feminina (3).

Um estudo investigou o conteúdo de chumbo no fluido ovariano folicular de 23 mulheres. Além disso, o efeito direto do chumbo sobre a morfologia e produção de progesterona por cultura de células granulosas de seis mulheres foi investigado num experimento in vitro. O grupo concluiu que os níveis de chumbo no fluido ovariano folicular pareciam não representar risco com respeito à secreção de progesterona pelo ovário (46).

Os efeitos do chumbo sobre o sistema reprodutor masculino em humanos não foram bem caracterizados. Os dados disponíveis apóiam uma con- clusão experimental de que os efeitos sobre os testículos, incluindo contagem reduzida de esperma e motilidade diminuída, podem resultar de exposição crônica com níveis de $\mathrm{Pb}-\mathrm{S}$ de $40 \mathrm{a}$ $50 \mu \mathrm{g} \cdot \mathrm{dL}^{-1}(4,7)$. Bonde et al. concluíram que são improváveis os efeitos adversos do chumbo sobre a função testicular em concentrações abaixo de $45 \mu \mathrm{g} . \mathrm{dL}^{-1}$ (47). De acordo com Marchlewicz, o chumbo é encontrado somente no citoplasma dos macrófagos no tecido intersticial, e atravessa a barreira hemato-epidídima (48). A eficácia terapêutica de uma combinação do ácido meso-2,3-dimercaptosuccínico (DMSA) com edetato de cálcio dissódico (EDTA) foi investigada em ratos, para a proteção das desordens testiculares na intoxicação por chumbo. Os resultados indicaram que a aplicação da terapia combinada durante dois períodos de 5 dias produziam uma recuperação mais efetiva nas desordens bioquímicas e histopatológicas induzidas pelo chumbo do que a terapia convencional de 5 dias apenas (49). Gandley et al. (50) estudaram o efeito da exposição a níveis relativamente baixos de acetato de chumbo (25 e 250 ppm) sobre a fertilidade e viabilidade de descendência em ratos machos. Esses autores concluíram que a fertilidade foi reduzida com níveis de $\mathrm{Pb}-\mathrm{S}$ na faixa de 27 a $60 \mu \mathrm{g} . \mathrm{dL}^{-1}$ e que a expressão genômica inicial foi afetada nos embriões cujos pais apresentavam concentrações de $\mathrm{Pb}-\mathrm{S}$ entre 15 e $23 \mu \mathrm{g} . \mathrm{dL}^{-1}$. Apesar de o polimorfismo genético do ácido deltaaminolevulínico desidratase parecer modificar a associação entre o $\mathrm{Pb}-\mathrm{S}$ e zinco protoporfirina, não foram encontradas alterações para coproporfirina urinária e contagem ou concentração de esperma em empregados de uma fundição de zinco-chumbo (51).

Há evidências qualitativas suficientes para apoiar a conclusão de que níveis elevados de exposição ao chumbo causam efeitos adversos significativos sobre a reprodução humana. Entretanto, os dados não permitem estimar que níveis de $\mathrm{Pb}-\mathrm{S}$ causariam danos em mulheres; em homens, essa definição é apenas especulativa $(4,52)$.

O chumbo também atravessa a barreira placentária e pode causar danos 
fetais $(1,2)$. Os dados encontrados por Nashashibi et al. sugerem uma correlação estatisticamente significativa entre a concentração de $\mathrm{Pb}-\mathrm{S}$ materna e a concentração de chumbo no cordão umbilical ou no leite, mostrando que há transferência do metal para o feto ou recém-nascido, respectivamente (53). Há fortes evidências de que o chumbo afeta não somente a viabilidade do feto, mas também seu desenvolvimento. As conseqüências da exposição pré-natal a baixos níveis de chumbo incluem peso reduzido ao nascer e nascimento prematuro (3-5, 7). Os experimentos com ratos demonstraram que um aumento de cálcio na dieta durante a gravidez pode reduzir a acumulação de chumbo fetal, mas não pode prevenir as reduções de peso e altura induzidas pelo $\mathrm{Pb}$ (54). Os estudos de Odland et al. (55) mostraram que o nível de chumbo no sangue materno é um fator preditivo de baixo peso de nascimento. Numa revisão da literatura epidemiológica, Andrews et al. (56) concluíram que o chumbo parece aumentar o risco de nascimento prematuro. Entretanto, os estudos de Sánchez et al. (57) sugerem que a exposição intrauterina ao chumbo pode estar associada com nascimento prematuro na primeira gravidez, mas não nas subseqüentes.

O chumbo é teratogênico em animais; entretanto, a maioria dos estudos em humanos não conseguiu mostrar uma relação entre os níveis de chumbo e as malformações congênitas $(3-5,7)$.

\section{Efeitos carcinogênicos}

A Agência Internacional de Pesquisa sobre o Câncer (International Agency for Research on Cancer, IARC) concluiu que as evidências relativas à carcinogenicidade do chumbo e seus compostos em seres humanos eram inadequadas. Entretanto, as provas dos efeitos carcinogênicos em animais eram suficientes. Assim, de acordo com a IARC, o chumbo inorgânico e os compostos de chumbo foram classificados como "possivelmente carcinogênicos para humanos" (grupo 2B) (3-5). Nos Esta- dos Unidos, uma lista das 20 substâncias mais perigosas para 2001 mostra o chumbo em segundo lugar, atrás somente do arsênio (58).

Os relatos de casos implicam o chumbo como potencial carcinogênico renal em humanos, mas a associação permanece incerta. Os sais solúveis, tais como o acetato e o fosfato de chumbo, têm sido apontados como causa de tumores em rins de ratos $(1,7)$.

Dados recentes indicam que o chumbo pode substituir o zinco em várias proteínas que funcionam como reguladoras da transcrição, incluindo as protaminas. Além disso, o chumbo reduz a ligação dessas proteínas com os elementos de identificação no DNA genômico. Isso sugere um envolvimento epigenético do chumbo na expressão do gene alterado. Esses eventos podem ser de particular relevância nas exposições transplacentárias e no câncer (59).

\section{Efeitos cardiovasculares}

Nas intoxicações agudas por chumbo, particularmente se o paciente tem cólica, a pressão sangüínea é freqüentemente elevada, podendo ocorrer também hipotonia e danos ao miocárdio. Os efeitos cardiovasculares crônicos do chumbo têm sido estudados, mas com resultados divergentes.

Considerando os estudos já realizados, parece que a exposição controlada ao chumbo no ambiente de trabalho não causa arteriosclerose, hipertensão, infarto do miocárdio ou doença cerebrovascular. As evidências de estudos clínicos, ocupacionais e com a população em geral sugerem que o chumbo afeta o sistema cardiovascular em humanos, produzindo lesões cardíacas, anormalidades eletrocardiográficas e aumentos na pressão sangüínea em níveis excessivos de exposição. Porém, a contribuição do chumbo, comparada com outros fatores que afetam a pressão do sangue, parece ser relativamente pequena, em torno de 1 a $2 \%$ da variação. Os dados animais demonstram claramente que o chumbo aumenta a pressão sangüínea sob condições experimentais controladas, e vários mecanismos têm sido propostos para explicar essas observações (1, 3-5).

Diversos estudos com grande número de trabalhadores expostos ao chumbo e na população em geral encontraram associação entre o metal e algum tipo de efeito sobre o sistema cardiovascular. Entretanto, todas as correlações positivas foram fracas e, também, uma relação causal não foi solidamente estabelecida. Os fatores de confundimento, tais como idade, sexo, álcool, fumo e exposição a múltiplos poluentes, entre outros, não foram controlados na maioria das avaliações (5).

Os dados sobre a relação entre o $\mathrm{Pb}-\mathrm{S}$ e a pressão sangüínea em estudos a epidemiológicos da população em geral permanecem controversos (60). Dolenc et al. (61) pesquisaram a possível influência de baixos níveis de exposição ao chumbo sobre a pressão sangüínea em 827 homens e 821 mulheres e concluíram que a exposição ao chumbo não está associada com o aumento da pressão sangüínea na população em geral. Nordberg et al. (18) chegaram a uma conclusão idêntica ao estudarem uma população de 804 idosos (média de idade $=88,4$ anos). No entanto, uma pesquisa realizada na Inglaterra com 4326 homens e mulheres acima de 16 anos encontrou uma associação positiva entre o chumbo no sangue e a pressão diastólica em homens (62).

Uma pesquisa com 798 trabalhadores ocupacionalmente expostos ao chumbo e 135 controles concluiu que o genótipo do receptor da vitamina $\mathrm{D}$, assim como o chumbo no sangue, na tíbia e no chumbo quelado ao DMSA, estava associado com a pressão sistólica, sendo que o chumbo na tíbia era o único biomarcador significativo do estado de hipertensão. Ao contrário do receptor da vitamina $\mathrm{D}$, o genótipo do ácido delta-aminolevulínico desidratase não estava associado à pressão e não modificou as associações das medidas de dose do chumbo com quaisquer das medidas de pressão do sangue (45).

Apesar dos esforços intensos para definir uma relação entre o conteúdo corpóreo de chumbo e a pressão do sangue ou outros efeitos sobre o sistema car- 
diovascular, nenhuma relação causal foi demonstrada em humanos (3).

\section{Efeitos gastrintestinais}

A cólica é um efeito inicial no quadro de intoxicação por chumbo em sujeitos ocupacionalmente expostos ou em indivíduos com exposição aguda a níveis elevados de chumbo, sendo também um sintoma de envenenamento por chumbo em crianças. Embora ocorra tipicamente em níveis de $\mathrm{Pb}-\mathrm{S}$ de 100 a $200 \mu \mathrm{g} \cdot \mathrm{dL}^{-1}$, também tem sido notada, algumas vezes, em trabalhadores com níveis mais baixos, tais como 40 a $60 \mu \mathrm{g} . \mathrm{dL}^{-1}(1,3-5,63,64)$.

Uma outra manifestação bem conhecida da exposição ao chumbo é a linha azulada nas gengivas (63). Entretanto, essa tão conhecida linha do chumbo não diz sequer se o paciente está intoxicado por chumbo. É formada por precipitado de sulfeto de chumbo e somente indica que o paciente tem estado exposto a esse metal e que, além disso, sua higiene dental é pobre $(1,5)$.

Embora os sintomas gastrintestinais sejam, há muito tempo, considerados característicos de envenenamento por chumbo, pouca atenção tem sido dada para a definição das relações de doseefeito. Esses sintomas ocorrem em trabalhadores expostos ao chumbo cuja exposição original é por inalação, e em crianças quando a exposição é pela via oral (4).

\section{Efeitos hepáticos}

Parece que não há novas evidências relacionando o conteúdo corpóreo de chumbo aos efeitos no fígado. Porém, há sugestões de que os efeitos do chumbo sobre a síntese da heme podem reduzir a capacidade funcional do citocromo P-450 do sistema hepático para metabolizar drogas, conforme demonstrado em trabalhadores ocupacionalmente expostos. Esses distúrbios no citocromo P-450 são mínimos no caso de intoxicação crônica pelo chumbo em adultos, mas significativos em crianças com envenenamento agudo $(3,5)$.
Hsiao et al. (65) avaliaram a relação entre os níveis de $\mathrm{Pb}-\mathrm{S}$ e indicadores hematológicos, hepáticos e renais em trabalhadores expostos ocupacionalmente e concluíram que a exposição por longo período ao chumbo estimula, contraditoriamente, a produção de células vermelhas e hematócrito, porém seu efeito sobre as funções hepática e renal não foi elucidado.

\section{CONCLUSÕES}

O chumbo afeta adversamente vários órgãos e sistemas, sendo que as alterações subcelulares e os efeitos neurológicos sobre o desenvolvimento parecem ser os mais críticos. Esse metal produz efeitos sobre muitos processos bioquímicos; em particular, afeta a síntese da heme, o sistema hematopoético e a homeostase do cálcio, interferindo em outros processos celulares. Embora os ossos sejam os maiores depósitos de chumbo do organismo, os dados disponíveis sobre seu efeito e mecanismo de ação nesses depósitos são poucos e inconclusivos.

As crianças são mais vulneráveis aos efeitos do chumbo por razões neurológicas, metabólicas e comportamentais. A encefalopatia é um dos mais sérios desvios tóxicos induzidos pelo chumbo em adultos e crianças, levando a disfunções psicológicas e neurocomportamentais. Os danos sobre o sistema nervoso periférico são encontrados principalmente em adultos.

$\mathrm{O}$ efeito do chumbo sobre o coração é indireto, ocorrendo por meio do sistema nervoso autônomo, sem efeito direto sobre o miocárdio. Apesar dos esforços para definir ligações entre o chumbo e os efeitos sobre o sistema cardiovascular, nenhuma relação causal tem sido demonstrada em humanos.

$\mathrm{O}$ chumbo causa nefropatia com disfunção tubular renal reversível, que ocorre, na maioria das vezes, em crianças sob exposição aguda, e nefropatia intersticial irreversível, efeito direto da exposição crônica sobre os rins, encontrada principalmente em trabalhadores expostos. A gota saturnínica é o resultado da função tubular reduzida, pois o chumbo interfere na excreção dos sais de ácido úrico.

Os efeitos reprodutivos do chumbo nos homens estão limitados à morfologia do esperma e à contagem. Nas mulheres, algumas conseqüências adversas na gravidez têm sido atribuídas ao chumbo. Como o chumbo atravessa a barreira placentária, pode causar danos fetais e, por isso, existem fortes evidências de que esse metal afeta não somente a viabilidade do feto, mas também seu desenvolvimento. Assim, o chumbo parece aumentar o risco de nascimento prematuro e parece reduzir o peso e altura ao nascer. Diversos estudos recentes sugerem que o crescimento em estatura pode ser afetado mesmo por exposição a níveis baixos de chumbo.

Quanto à teratogenicidade e carcinogenicidade, os dados são inadequados para demonstrar uma associação entre o chumbo e tais efeitos em humanos.

Os efeitos gastrintestinais ocorrem basicamente em sujeitos ocupacionalmente expostos ou em indivíduos com exposição aguda a níveis elevados de chumbo, porém são poucas as pesquisas sobre esse assunto. A cólica é um efeito precoce consistente de intoxicação por chumbo, enquanto que a anemia, apesar de ser uma descoberta extraordinária no envenenamento por chumbo, não é uma manifestação precoce, sendo rara sem outros efeitos detectáveis, e só é evidente se os níveis de $\mathrm{Pb}-\mathrm{S}$ forem elevados por períodos prolongados.

Parece não haver evidências que relacionem o chumbo com efeitos no fígado, porém há sugestões de que os efeitos desse metal sobre a síntese da heme podem alterar a capacidade funcional do sistema citocromo P-450 hepático para metabolizar drogas. Por outro lado, diversos estudos concluíram que a interferência do chumbo sobre a síntese da heme pode ser também a base dos efeitos sobre o metabolismo da vitamina D. Entretanto, as indicações de danos endócrinos sobre os eixos hipotálamo-pituitária-tireóide/ supra-renal pela exposição ao chumbo em humanos devem ser vistas com cautela. 
Apesar de os estudos científicos sobre a toxicologia do chumbo virem sendo desenvolvidos há mais de um século, há muitas questões ainda sem solução. São insuficientes as informações sobre os mecanismos de ação que originam os efeitos tóxicos do chumbo. Logo, as pesquisas continuam sendo necessárias para o completo entendimento e, conseqüentemente, a prevenção desses efeitos.

Os resultados da exposição ao chumbo sobre os ossos, os sistemas nervoso central e cardiovascular, os rins e o fígado devem ser estudados com maior profundidade, bem como os efeitos sobre a reprodução masculina e feminina, o sistema endócrino e a formação do feto. Também é essencial esclarecer se o chumbo tem efeitos teratogênicos e carcinogênicos em seres humanos.

\section{REFERÊNCIAS}

1. Saryan LA, Zenz C. Lead and its compounds. Em: Zenz OC, Dickerson B, Horvath EP, eds. Occupational medicine. $3^{\underline{a}}$ ed. St. Louis: Mosby-Year Book; 1994. Pp. 506-541.

2. Goyer RA. Toxic effects of metals - lead. Em: Amdur MO, Dull J, Klaassen CD, eds. Casareh and Doull's toxicology - the basic science of poisons. $4^{\mathrm{a}}$ ed. New York: Pergamon Press; 1991. Pp. 639-646.

3. World Health Organization. Environmental health criteria 165: inorganic lead. Em: IPCS (International Programme on Chemical Safety). Geneva: WHO; 1995.

4. US Department of Health and Human Services, Public Health Service, ATSDR (Agency for Toxic Substances and Disease Registry). Toxicological profile for lead. Atlanta, Georgia: U.S. Department of Health and Human Services; 1999.

5. Skerfving S. Inorganic lead. Em: Beije B, Lundberg P, eds. Criteria documents from the Nordic Expert Group 1992. Stockholm: Arbete och Hälsa; 1993. Pp. 125-238.

6. Tsalev DL, Zaprianov ZK. Lead. Em: Atomic absorption spectrometry in occupational and environmental health practice. $2^{\mathrm{a}}$ ed. Florida: CRC Press; 1985. Pp. 137-150.

7. US Department of Health and Human Services, Public Health Service, ATSDR (Agency for Toxic Substances and Disease Registry). Case studies in environmental medicine: lead toxicity. Atlanta, Georgia: U.S. Department of Health and Human Services; 1992.

8. Adachi JD, Arlen D, Webber CE, Chettle DR, Beaumont LF, Gordon CL. Is there any association between the presence of bone disease and cumulative exposure to lead? Calcif Tissue Int. 1998;63(5):429-432.

9. Elinder CG, Friberg L, Kjellström T, Nordberg $\mathrm{G}$, Oberdoerster G. Biological monitoring of metals. Em: The global environmental epidemiology network. Geneva: World Health Organization; 1994.

10. De Gennaro LD. Lead and the developing nervous system. Growth Dev Aging. 2002; 66(1):43-50.

11. Lanphear BP, Dietrich K, Auinger P, Cox C. Cognitive deficits associated with blood lead concentrations $<10$ microg/dL in US children and adolescents. Public Health Rep. 2000; 115(6):521-529.

12. Stewart WF, Schwartz BS, Simon D, Kelsey K, Todd AC. ApoE genotype, past adult lead ex- posure, and neurobehavioral function. Environ Health Perspect. 2002;110(5):501-505.

13. Shen $X, W u S$, Yan C. Impacts of low-level lead exposure on development of children: recent studies in China. Clin Chim Acta. 2001; 313(1-2):217-220.

14. Osterberg K, Borjesson J, Gerhardsson L, Schutz A, Skerfving S. A neurobehavioural study of long-term occupational inorganic lead exposure. Sci Total Environ. 1997;201(1): 39-51.

15. Hanninen $\mathrm{H}$, Aitio A, Kovala $\mathrm{T}$, Luukkonen R, Matikainen E, Mannelin T, et al. Occupational exposure to lead and neuropsychological dysfunction. Occup Environ Med. 1998; 55(3):202-209.

16. Schwartz BS, Stewart WF, Bolla KI, Simon PD, Bandeen-Roche K, Gordon PB, et al. Past adult lead exposure is associated with longitudinal decline in cognitive function. Neurology. 2000;55(8):1144-1150.

17. Kumar P, Husain SG, Murthy RC, Srivastava SP, Anand M, Ali MM, et al. Neuropsychological studies on lead battery workers. Vet Hum Toxicol. 2002;44(2):76-78.

18. Nordberg M, Winblad B, Fratiglioni L, Basun $\mathrm{H}$. Lead concentrations in elderly urban people related to blood pressure and mental performance: results from a population-based study. Am J Ind Med. 2000;38(3):290-294.

19. Rubens O, Logina I, Kravale I, Eglite M, Donaghy M. Peripheral neuropathy in chronic occupational inorganic lead exposure: a clinical and electrophysiological study. J Neurol Neurosurg Psychiatry. 2001;71(2):200-204.

20. Murata K, Araki S, Yokoyama K, Uchida E, Fujimura Y. Assessment of central, peripheral, and autonomic nervous system functions in lead workers: neuroelectrophysiological studies. Environ Res. 1993;61(2):323-336.

21. Hirata M, Kosaka H. Effects of lead exposure on neurophysiological parameters. Environ Res. 1993;63(1):60-69.

22. Murata K, Araki S, Yokoyama K, Nomiyama K, Nomiyama H, Tao YX, et al. Autonomic and central nervous system effects of lead in female glass workers in China. Am J Ind Med. 1995;28(2):233-244.

23. Araki S, Sato H, Yokoyama K, Murata K. Subclinical neurophysiological effects of lead: a review on peripheral, central, and autonomic nervous system effects in lead workers. Am J Ind Med. 2000;37(2):193-204
24. Kovala T, Matikainen E, Mannelin T, Erkkila J, Riihimaki V, Hanninen $\mathrm{H}$, et al. Effects of low level exposure to lead on neurophysiological functions among lead battery workers. Occup Environ Med. 1997;54(7):487-493.

25. $\mathrm{Hu} \mathrm{H}$, Watanabe $\mathrm{H}$, Payton M, Korrick S, Rotnitzky A. The relationship between bone lead and hemoglobin. JAMA. 1994;272(19): 1512-1517.

26. Jacob B, Ritz B, Heinrich J, Hoelscher B, Wichmann HE. The effect of low-level blood lead on hematologic parameters in children. Environ Res. 2000;82(2):150-159.

27. Serwint JR, Damokosh AI, Berger OG, Chisolm JJJR, Gunter EW, Jones RL, et al. No difference in iron status between children with low and moderate lead exposure. J Pediatr. 1999;135(1):108-110.

28. Wright RO. The role of iron therapy in childhood plumbism. Curr Opin Pediatr. 1999; 11(3):255-258.

29. Osterode W, Barnas U, Geissler K. Dose dependent reduction of erythroid progenitor cells and inappropriate erythropoietin response in exposure to lead: new aspects of anaemia induced by lead. Occup Environ Med. 1999;56(2):106-109.

30. Counter SA, Buchanan LH, Ortega F, Rifai N. Blood lead and hemoglobin levels in Andean children with chronic lead intoxication. Neurotoxicology. 2000;21(3):301-308.

31. Suplido ML, Ong CN. Lead exposure among small-scale battery recyclers, automobile radiator mechanics, and their children in Manila, the Philippines. Environ Res. 2000;82(3):231238.

32. Vahter M, Counter SA, Laurell G, Buchanan LH, Ortega F, Schutz A, et al. Extensive lead exposure in children living in an area with production of lead-glazed tiles in the Ecuadorian Andes. Int Arch Occup Environ Health. 1997;70(4):282-286.

33. Froom P, Kristal-Boneh E, Benbassat J, Ashkanazi R, Ribak J. Lead exposure in battery-factory workers is not associated with anemia. J Occup Environ Med. 1999;41(2): $120-123$.

34. Koo WW, Succop PA, Bornschein RL, KrugWispe SK, Steinchen JJ, Tsang RC, et al. Serum vitamin $\mathrm{D}$ metabolites and bone mineralization in young children with chronic low to moderate lead exposure. Pediatrics. 1991; 87(5):680-687. 
35. Singh B, Chandran V, Bandhu HK, Mittal BR, Bhattacharya A, Jindal SK, et al. Impact of lead exposure on pituitary-thyroid axis in humans. Biometals. 2000;13(2):187-192.

36. Erfurth EM, Gerhardsson L, Nilsson A, Rylander L, Schutz A, Skerfving S, et al. Effects of lead on the endocrine system in lead smelter workers. Arch Environ Health. 2001; 56(5):449-455.

37. Ballew C, Khan LK, Kaufmann R, Mokdad A, Miller DT, Gunter EW. Blood lead concentration and children's anthropometric dimensions in the Third National Health and Nutrition Examination Survey (NHANES III), 1988-1994. J Pediatr. 1999;134(5):623-630.

38. Berglund M, Akesson A, Bjellerup P, Vahter M. Metal-bone interactions. Toxicol Lett. 2000; 112-113:219-225.

39. Vivoli G, Fantuzzi G, Bergomi M, Tonelli E, Gatto MR, Zanetti F, et al. Relationship between low lead exposure and somatic growth in adolescents. J Exp Anal Environ Epidemiol. 1993;3(Suppl.1):201-209.

40. Hicks DG, O'Keefe RJ, Reynolds KJ, CorySlechta DA, Puzas JE, Judkins A. Effects of lead on growth plate chondrocyte phenotype. Toxicol Appl Pharmacol. 1996;140(1):164-172.

41. Zuscik MJ, Pateder DB, Puzas JE, Schwarz EM, Rosier RN, O'Keefe RJ. Lead alters parathyroid hormone-related peptide and transforming growth factor-beta1 effects and AP-1 and NF-kappaB signaling in chondrocytes. J Orthop Res. 2002;20(4):811-818.

42. Mortada WI, Sobh MA, El-Defrawy MM, Farahat SE. Study of lead exposure from automobile exhaust as a risk for nephrotoxicity among traffic policemen. Am J Nephrol. 2001;21(4):274-279.

43. Loghman-Adham M. Renal effects of environmental and occupational lead exposure. Environ Health Perspect. 1997;105(9):928-939.

44. Nolan CV, Shaikh ZA. Lead nephrotoxicity and associated disorders: biochemical mechanisms. Toxicology. 1992;73(2):127-146.

45. Lee BK, Lee GS, Stewart WF, Ahn KD, Simon D, Kelsey KT, et al. Associations of blood pressure and hypertension with lead dose measures and polymorphisms in the vitamin $D$ receptor and delta-aminolevulinic acid dehydratase. Environ Health Perspect. 2001; 109(4):383-389.

46. Paksy K, Gati I, Naray M, Rajczy K. Lead accumulation in human ovarian follicular fluid, and in vitro effect of lead on progesterone production by cultured human ovarian granulosa cells. J Toxicol Environ Health. 2001; 62(5):359-366

47. Bonde JP, Joffe M, Apostoli P, Dale A, Kiss P, Spano M, et al. Sperm count and chromatin structure in men exposed to inorganic lead: lowest adverse effect levels. Occup Environ Med. 2002;59(2):234-242.

48. Marchlewicz M. Effectiveness of blood-testis and blood-epididymis barriers for lead. Ann Acad Med Stetin. 1994;40:37-51.

49. Flora GJ, Arora U, Seth PK. Influence of combined therapeutic potential of meso 2,3dimercaptosuccinic acid and calcium disodium edetate on lead-induced testicular alterations in rats. Biomed Environ Sci. 1999; 12(4):285-291.

50. Gandley R, Anderson L, Silbergeld EK. Lead: male-mediated effects on reproduction and development in the rat. Environ Res. 1999; 80(4):355-363.

51. Alexander $\mathrm{BH}$, Checkoway $\mathrm{H}$, Costa-Mallen P, Faustman EM, Woods JS, Kelsey KT, et al. Interaction of blood lead and $\delta$-aminolevulinic acid dehydratase genotype on markers of heme synthesis and sperm production in lead smelter. Environ Health Perspect. 1998;106(4):213-216.

52. Alexander $\mathrm{BH}$, Checkoway $\mathrm{H}$, van Netten $\mathrm{C}$, Muller CH, Ewers TG, Kaufman JD, et al. Semen quality of men employed at a lead smelter. Occup Environ Med. 1996;53(6):411416.

53. Nashashibi N, Cardamakis E, Bolbos G, Tzingounis $V$. Investigation of kinetic of lead during pregnancy and lactation. Gynecol Obstet Invest. 1999;48(3):158-162.

54. Han SG, Pfizenmaier DH, Garcia E, Eguez ML, Ling M, Kemp FW. Effects of lead exposure before pregnancy and dietary calcium during pregnancy on fetal development and lead accumulation. Environ Health Perspect. 2000;108(6):527-531.

55. Odland JO, Nieboer E, Romanova N, Thomassen Y, Lund E. Blood lead and cadmium and birth weight among sub-arctic and arctic populations of Norway and Russia. Acta Obstet Gynecol Scand. 1999;78(10):852860.

56. Andrews KW, Savitz DA, Hertz-Picciotto I. Prenatal lead exposure in relation to gestational age and birth weight: a review of epi- demiologic studies. Am J Ind Med. 1994;26(1): 13-32.

57. Torres-Sánchez LE, Berkowitz G, Carrillo LL, Arreola LT, Rios C, Cervantes MC. Intrauterine lead exposure and preterm birth. Environ Res. 1999;81(4):297-301.

58. US Department of Health and Human Services, Public Health Service, ATSDR (Agency for Toxic Substances and Disease Registry). Top 20 hazardous substances, ATSDR/EPA Priority List for 2001. Disponível em: http:/ www.atsdr.cdc.gov/cxcx3.html. Acessado em 14 de janeiro de 2002.

59. Silbergeld EK, Waalkes M, Rice JM. Lead as a carcinogen: experimental evidence and mechanisms of action. Am J Ind Med. 2000;38(3): 316-323.

60. Victery W, Tyroler HA, Volpe R, Grant LD. Summary of discussion sessions: symposium on lead-blood pressure relationships. Environ Health Perspect. 1988;78:139-155.

61. Dolenc P, Staessen JA, Lauwerys RR, Amery A. Short report: low-level lead exposure does not increase the blood pressure in the general population. Cadmibel Study Group. J Hypertens. 1993;11(5):589-593.

62. Bost L, Primatesta P, Dong W, Poulter N. Blood lead and blood pressure: evidence from the Health Survey for England 1995. J Hum Hypertens. 1999;13(2):123-128.

63. Tandon SK, Chatterjee M, Bhargava A, Shukla $\mathrm{V}$, Bihari V. Lead poisoning in Indian silver refiners. Sci Total Environ. 2001;281(1-3):177182.

64. Popp W, Werfel U, Peters T, Kramer R, Bruch J. Occupational lead poisoning due to deficient protective measures at the work place. Dtsch Med Wochenschr. 2001;126(43):12011204.

65. Hsiao CY, Wu HD, Lai JS, Kuo HW. A longitudinal study of the effects of long-term exposure to lead among lead battery factory workers in Taiwan (1989-1999). Sci Total Environ. 2001;279(1-3):151-158.

Manuscrito recebido em 24 de outubro de 2002. Aceito em versão revisada el 1 de outubro de 2003. 
ABSTRACT Objective. To review the literature concerning the risks associated with exposure to lead and lead compounds, especially in children and in populations that are occupationally exposed.

\section{Effects of lead exposure on the human body and health implications}

Data sources. Using "chumbo" [lead] and "efeitos" [effects] as search terms, two large databases, namely PubMed (United States National Library of Medicine) and LILACS (Literatura Latino-Americana e do Caribe em Ciências da Saúde [Latin American and Caribbean Literature in the Health Sciences]), were searched for studies on lead toxicity from 1988 to 2002. Other sources used to conduct the search include the web page of the United States Agency for Toxic Substances and Disease Registry, in Atlanta, Georgia, and the library of the Toxicology Laboratory of the Center for Workers' Health and Human Ecology at the National School of Public Health [Centro de Estudos da Saúde de Trabalhador e Ecologia Humana, Escola Nacional de Saúde Pública], Fundação Oswaldo Cruz, Rio de Janeiro, Brazil.

Conclusions. The toxic effects of lead and lead compounds have been extensively studied for over a century. In recent years, epidemiologic studies have focused primarily on the neurotoxic effects of lead on children, particularly in terms of impaired intellectual ability and behavioral problems. However, there is still insufficient information on the mechanisms of action that account for such toxicity. More in-depth studies are also needed on the effects of lead exposure on bone, the central nervous system, the cardiovascular system, the kidneys, the liver, the male and female reproductive systems, and the endocrine system. The potential teratogenicity and carcinogenicity of lead, as well as its effect on pregnancy outcomes and neonatal growth and development, also require further study.

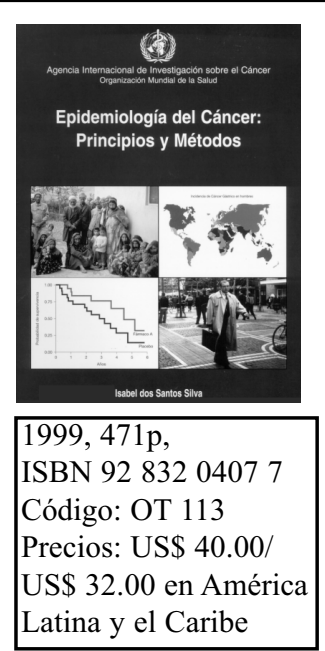

Epidemiología del cáncer: principios y métodos

Esta obra, que ya está disponible por medio de la OPS, se dirige principalmente a estudiantes de medicina y salud pública, médicos, profesionales de la salud y cualquiera interesado en entender los principios y métodos aplicados para estudiar la epidemiología del cáncer.

La obra está diseñada para proporcionar --mediante ejemplos ilustrativos y, con frecuencia, datos derivados de investigaciones-- información fácilmente comprensible sobre los conceptos básicos y métodos de investigación en el campo de la epidemiología. Dado que algunos conocimientos de estadística son indispensables para realizar, analizar e interpretar los estudios epidemiológicos, también se presentan fórmulas y conceptos estadísticos, aunque se hace mayor hincapié en la interpretación de los datos que en los cálculos en sí.

Usted también puede enviar su pedido y pago en dólares estadounidenses a: http://publications.paho.org Fax: (301) 206-9789・E-mail: paho@pmds.com・Oficina de la OPS/OMS en su país 